\title{
SAP97-mediated local trafficking is altered in Alzheimer disease patients' hippocampus
}

\author{
Elena Marcello ${ }^{\mathrm{a}, 1}$, Roberta Epis ${ }^{\mathrm{a}, 1}$, Claudia Saraceno $^{\mathrm{a}}$, Fabrizio Gardoni ${ }^{\mathrm{a}}$, Barbara Borroni ${ }^{\mathrm{b}}$, \\ Flaminio Cattabeni ${ }^{\mathrm{a}}$, Alessandro Padovani ${ }^{\mathrm{b}}$, Monica Di Luca ${ }^{\mathrm{a}, *}$ \\ ${ }^{a}$ Università degli Studi di Milano, Department of Pharmacological Sciences and Centre of Excellence on Neurodegenerative Diseases, Milan, Italy \\ ${ }^{b}$ Department of Neurological Sciences, University of Brescia, Brescia, Italy \\ Received 9 March 2010; received in revised form 26 July 2010; accepted 16 September 2010
}

\begin{abstract}
Synapse-asssociated protein-97 (SAP97) is responsible for the trafficking of both glutamate receptor subunits, GluR1 and NR2A, and $\alpha$-secretase ADAM10 to the synaptic membrane. Here we evaluate the trafficking capability of SAP97 in Alzheimer disease (AD) patients' brain. We analyzed autoptic hippocampus and superior frontal gyrus, respectively as an affected and a less affected area, from 6 AD patients (Braak 4) and 6 healthy controls. In hippocampus, but not in superior frontal gyrus, of AD patients, ADAM10 and GluR1 synaptic membrane levels are altered while NR2A localization is not affected. Both immunoprecipitation and pull-down assays demonstrated that SAP97 failed to correctly couple to ADAM10 and GluR1, but not to NR2A. These findings not only indicate SAP97 as a point of convergence between amyloid cascade and synaptic failure in $\mathrm{AD}$, but also allow a different interpretation of $\mathrm{AD}$ which can be now perceived as synaptic trafficking defect pathology.
\end{abstract}

(C) 2012 Elsevier Inc. All rights reserved.

Keywords: Alzheimer Disease; Scaffolding proteins; ADAM10; AMPA receptor; NMDA receptor; Glutamatergic synapse

\section{Introduction}

Alzheimer disease (AD) is the most common form of dementia. Neuropathology of the full blown stage of the disease reveals the presence of both amyloid aggregates and neurofibrillary tangles, indicated as hallmarks of the disease. In addition, quantification by both immunostaining and electron microscopy of synaptic markers documented significant decrease in synaptic density in both cortex and hippocampus of AD patients' brains and biochemical analyses confirmed a similar loss in both pre- and postsynaptic components (Bertoni-Freddari et al., 1996, 2003; DeKosky and Scheff, 1990; Masliah et al., 2001; Reddy et al., 2005; Scheff et al., 2007; Terry et al., 1991). This initial decrease

\footnotetext{
* Corresponding author at: Department of Pharmacological Sciences, University of Milano, Via Balzaretti 9, 20133 Milano, Italy. Tel.: 003902 50318374; fax: 00390250318284.

E-mail address: monica.diluca@unimi.it (M. Di Luca).

${ }^{1}$ These 2 authors contributed equally to this work.
}

of synaptic contacts seems not to correlate with loss of neuronal cell bodies thus suggesting synaptic failure and subsequent synaptic loss as a very early event in $\mathrm{AD}$ pathogenesis (Bertoni-Freddari et al., 2003; DeKosky and Scheff, 1990).

More recently it has been proposed that the complex cascade of molecular events eventually leading to synaptic failure is the consequence of progressive accumulation and aggregation of amyloid $\beta(\mathrm{A} \beta$ ) peptide (Hardy and Selkoe, 2002). However, cellular and molecular targets of $\mathrm{A} \beta$ and/or early molecular events initiating the process of synaptic impairment are still unknown.

$\mathrm{A} \beta$ peptide is located within the type I transmembrane amyloid precursor protein (APP) at the junction between the intralumenal and transmembrane domains. Two enzymatic steps liberate A $\beta$ from APP. In the first, " $\beta$-cleavage"” step, $\beta$-site APP-cleaving enzyme (BACE) (Vassar, 2001) cleaves APP at or near the N-terminus of the A $\beta$ peptide; then, in the second, or " $\gamma$-cleavage"" step, the membrane- 
bound C-terminal APP fragment (CTF99) generated by BACE goes on to be cleaved by the $\gamma$-secretase. Alternatively, APP can be subject to the $\alpha$-secretase cleavage mediated by $\mathrm{A}$ disintegrin and metalloproteinase 10 (ADAM10) (Lammich et al., 1999; Postina et al., 2004), which occurs within the sequence of $\mathrm{A} \beta$, thus precluding the formation of the amyloidogenic fragments.

ADAM10 is synthesized in an inactive form, which is proteolytically cleaved during its forward transport along the secretory pathway, enabling enzyme activity at the plasma membrane (Anders et al., 2001; Lammich et al., 1999).

We have previously reported that ADAM10 binds synapse-associated protein-97 (SAP97) and this direct interaction governs ADAM10 recruitment to synaptic membrane and its activity. This mechanism represents a novel pathway of activation of $\alpha$-secretase physiological APP cleavage in neurons at synaptic sites (Marcello et al., 2007).

SAP97 belongs to the membrane-associated guanylate kinase (PSD-MAGUK) family of synaptic scaffolding proteins which are key players in the structural organization of the excitatory glutamatergic synapse (Kim and Sheng, 2004). Some members of this family, i.e., PSD95/93 are in fact involved in spines' maturation, clustering of ionotropic glutamate receptors subunits at synaptic sites, while SAP102 and SAP97 have been shown to be essential partners for local synaptic trafficking of ionotropic glutamate receptors' subunits, i.e., amino-3-hydroxy-5-methylisoxazole-4-propionic acid (AMPA) and N-methyl-D-aspartic acid (NMDA) subunits (Elias and Nicoll, 2007). In particular, SAP97 is implicated in the precise targeting and clustering of AMPA receptor subunits GluR1 (Nakagawa et al., 2004; Sans et al., 2001; Schluter et al., 2006) and NMDA receptor subunit NR2A (Gardoni et al., 2003; Mauceri et al., 2007) through PDZ1 and 2 domains. Because of SAP97 involvement in trafficking of relevant molecules to the synapse, its role in neurodegenerative diseases has been largely addressed (Nash et al., 2005; Sato et al., 2008; Wakabayashi et al., 1999).

Moreover, an emerging concept in neuroscience is that activity dependent regulation of local trafficking of different synaptic proteins represents the basis of plasticity and, when dysfunctional, of synaptic failure occurring in many neurodegenerative diseases.

Here we explored the possibility that a defect of SAP97 capability to traffic synaptic proteins, both glutamate receptors subunits and $\alpha$-secretase ADAM10, could represent one of the initial molecular events leading to synaptic failure in $\mathrm{AD}$, eventually merging amyloid cascade and synaptic failure.

\section{Methods}

\subsection{Characteristics of the subjects}

The hippocampus (Hp) and the superior frontal gyrus (SFG) from late onset/sporadic $\mathrm{AD}$ patients (AD; $n=6$ ) and healthy controls ( $\mathrm{HC} ; n=6$ ) were obtained from the Netherlands Brain Bank. Stringent criteria were used in the case selection of human brain tissues employed in this study. HC have no history of psychiatric or neurological disease and no evidence of significant age-related neurodegeneration. Braak and Braak criteria were used to categorize AD tissues in accordance with established guidelines (Braak and Braak, 1991). As previously reported, these criteria are based on the distribution of neurofibrillary tangles (NFTs), which are seen first in the transentorhinal area (stage 1), spread to the entorhinal region (stage 2), extend to the hippocampus proper (stage 3 ), increase in number there (stage 4), and involve the association neocortex (stage 5), and finally the primary cortex (stage 6). These stages are divided into the entorhinal (stage 1-2), limbic (stage 3-4), and neocortical (stage 5-6) phase corresponding, respectively, to cognitively normal, cognitively impaired, and demented patients. Demographic and clinical characteristics

Table 1

Demographic and neuropathological characteristics of AD and HC cases

\begin{tabular}{|c|c|c|c|c|c|c|}
\hline Subjects & Gender & Age at death, years & PMD, hours & $\mathrm{pH} \mathrm{CSF}$ & Brain weight, $\mathrm{g}$ & Braak level \\
\hline 1 (AD) & $\mathrm{F}$ & 91 & 3.45 & 6.36 & 1011 & 4 \\
\hline $2(\mathrm{AD})$ & $\mathrm{F}$ & 91 & 4.15 & 6.27 & 1202 & 4 \\
\hline 3 (AD) & $\mathrm{F}$ & 86 & 4.10 & 6.34 & 1083 & 4 \\
\hline 4 (AD) & $\mathrm{F}$ & 86 & 5.05 & 6.62 & 998 & 4 \\
\hline 5 (AD) & M & 81 & 4.05 & 6.42 & 1253 & 4 \\
\hline $6(\mathrm{AD})$ & $\mathrm{F}$ & 86 & 5.55 & 6.85 & 950 & 4 \\
\hline Mean $\pm \mathrm{SD}$ & & $86.83 \pm 4.18$ & $4.39 \pm 0.57$ & $6.48 \pm 0.13$ & $1082.83 \pm 114$ & \\
\hline $1(\mathrm{HC})$ & $\mathrm{F}$ & 84 & 4.45 & 6.26 & 1179 & 1 \\
\hline $2(\mathrm{HC})$ & $\mathrm{F}$ & 81 & 6.40 & 7.16 & 1164 & 1 \\
\hline $3(\mathrm{HC})$ & M & 80 & 7.15 & 5.8 & 1376 & 0 \\
\hline $4(\mathrm{HC})$ & M & 84 & 7.05 & 5.9 & 1385 & 1 \\
\hline $5(\mathrm{HC})$ & $\mathrm{F}$ & 85 & 5.00 & 6.72 & 1257 & 1 \\
\hline $6(\mathrm{HC})$ & $\mathrm{F}$ & 85 & 4.40 & 6.71 & 1165 & 2 \\
\hline Mean $\pm \mathrm{SD}$ & & $83.17 \pm 2.17$ & $5.74 \pm 1.22$ & $6.43 \pm 0.57$ & $1254.33 \pm 105.03$ & \\
\hline
\end{tabular}

AD, Alzheimer's disease; CSF, cerebrospinal fluid; F, female; HC, healthy controls; M, male; PMD, postmortem delay. 
of $\mathrm{AD}$ patients and $\mathrm{HC}$ are shown in Table 1. AD patients fulfilled Braak 4 stage. Accordingly, in AD cases there were tangles and neuritic plaques in $\mathrm{Hp}$, whereas the amount of plaques in SFG closely resembled HC. All procedures were in accordance with the National Institutes of Health (NIH) Guide for the Care and Use of Laboratory human tissue and were approved by the Ethics Committee of the University of Milan, Italy.

\subsection{Triton insoluble synaptic membrane (TIF) and soluble fraction $(S 2)$ preparation}

Triton insoluble fraction (TIF), a fraction highly enriched in all categories of postsynaptic density proteins (i.e., receptor, signaling, scaffolding, and cytoskeletal elements) absent of presynaptic markers, and soluble fraction S2, were obtained as previously described (Gardoni et al., 2001; Marcello et al., 2007). In order to avoid protein degradation, AD samples were paired to $\mathrm{HC}$ samples and processed at the same time. The relative values for each case were replicated in duplicate experiments, considering for each paired sample the ratio $\mathrm{AD} / \mathrm{HC}$.

\subsection{Immunoprecipitation (i.p.)}

Aliquots of $50 \mu \mathrm{g}$ (for ADAM10/SAP97 and GluR1/ SAP97 immunoprecipitation [i.p.]) or of $70 \mu \mathrm{g}$ (for SAP97/ NR2A i.p.) of homogenate obtained from the $\mathrm{S} 2$ purification from $\mathrm{Hp}$ and $\mathrm{SFG}$ of $\mathrm{HC}$ and $\mathrm{AD}$ were incubated overnight at $4{ }^{\circ} \mathrm{C}$ in a buffer containing $200 \mathrm{mM} \mathrm{NaCl}, 10 \mathrm{mM}$ ethylene-diaminetetra-acetic acid (EDTA), $10 \mathrm{mM}$ $\mathrm{Na}_{2} \mathrm{HPO}_{4}, 0.5 \%$ NP-40, $0.1 \%$ sodium dodecyl sulfate (SDS) in a final volume of $150 \mu \mathrm{L}$ with an antibody (Ab) against ADAM10, GluR1, or SAP97. Immunoprecipitation has been carried out as previously described (Marcello et al., 2007). Beads were collected by centrifugation and the supernatants were loaded onto sodium dodecyl sulfate polyacrylamide gel electrophoresis (SDS-PAGE) at $6 \%$ and revealed by either anti-SAP97 or anti-NR2A.

\subsection{Western blotting (WB)}

$30 \mu \mathrm{g}$ of proteins from homogenate and TIF were separated on $6 \%$ and $7 \%$ SDS-PAGE, transferred to a nitrocellulose membrane and probed with the corresponding primary $\mathrm{Ab}$, followed by incubation with horseradish peroxidase-conjugated secondary $\mathrm{Ab}$. To analyze APP Cterminal fragments (CTFs), TIF proteins were separated on a $15 \%$ Tris-Tricine SDS-PAGE and Western blot (WB) analysis was performed with 4G8 Ab. Membranes were developed using electrochemiluminescence reagent (Amersham Biosciences, Buckinghamshire, UK).

\subsection{Cloning, expression, and purification of glutathione S-transferase (GST) fusion protein}

Glutathione S-transferase (GST)-ADAM10 C-terminal domain $(\mathrm{Ct})$ fusion protein contains the cytoplasmic domain of ADAM10 (695-749) (Marcello et al., 2007) and GST-
NR2A the sequence of NR2A C-terminal domain from amino acid 1349-1464 (Gardoni et al., 1999). GST-GluR1 C-terminal domain $(\mathrm{Ct})$ was kindly provided by M. Passafaro (DTI Dulbecco Telethon Institute, Milano, Italy) and includes the sequence of GluR1 cytoplasmic tail. GST fusion proteins were expressed in Escherichia coli and purified on glutathione agarose beads (Sigma Aldrich, St. Louis, MO, USA) as previously described (Gardoni et al., 2001).

\subsection{Pull-down assay}

Aliquots of homogenate from either $\mathrm{Hp}$ or SFG of $\mathrm{AD}$ patients and $\mathrm{HC}$ subjects were diluted with Tris Buffered Saline $(10 \mathrm{mM}$ Tris and $150 \mathrm{mM} \mathrm{NaCl})$ to a final volume of $1 \mathrm{~mL}$ and incubated 2 hours with $40 \mu \mathrm{L}$ of GST saturated with the C-terminal domain of ADAM10 or the C-terminal domain of NR2A or the C-terminal domain of GluR1 or GST alone. After incubation, beads were washed 5 times with Tris-buffered saline and $0.1 \%$ Triton X-100. Bound proteins were resolved by SDS-PAGE and subjected to immunoblot analysis with monoclonal SAP97 Ab and monoclonal GST Ab.

\subsection{Antibodies}

The following $\mathrm{Ab}$ were used: monoclonal antibody (mAb) 22C11 (raised against N-terminal domain of APP), mAb 4G8 (against 17-24 amino acids of $\mathrm{A} \beta$ ), mAb anti- $\alpha$-calcium/calmodulin-dependent kinase II $(\alpha$-CaMKII $)$, polyclonal antibody (pAb) anti-GluR1 and mAb 6E10, raised against 1-17 amino acids of $\mathrm{A} \beta$, were purchased from Millipore (Billerica, MA, USA); pAb anti-ADAM10 from Abcam (Cambridge, MA, USA); mAb antipan-Shank and anti GST from Neuromab (Davis, CA, USA); mAb anti-SAP97 from Stressgen (Victoria, B.C., Canada); mAb anti-PSD-95 from Affinity BioReagents, Inc. (Golden, CO, USA); pAb antiactin from Sigma-Aldrich (St. Louis, MO, USA); mAb against NR2A from Zymed (San Francisco, CA, USA); anti-synaptophysin and anti-SNAP25 from Synaptic Systems (Göttingen, Germany). Peroxidase-conjugated secondary anti-mouse $\mathrm{Ab}$ was purchased from Pierce (Rockford, IL, USA) while peroxidase-conjugated secondary anti-rabbit $\mathrm{Ab}$ was purchased from Bio-Rad (Hercules, CA, USA).

\subsection{Statistical analysis}

The levels of $\alpha$-CaMKII, PSD-95, Shank, SAP97, ADAM10, NR2A, and GluR1 were expressed as relative optical density (OD) measurements, i.e., ratio between the immunoreactivity of the protein of interest and the immunoreactivity of actin in each lane scanned. Data obtained by pull-down assays were normalized on GST OD, corresponding to the amount of GST fusion protein. The values for $\mathrm{AD}$ patients were then expressed as percentage of $\mathrm{HC}$ in the same blot \pm standard error of the mean (SEM). For the analysis of CTFs, the ratio between the OD of CTF83 and CTF99 was measured. Statistical evaluations were performed by Student $t$ test or Pearson correlations, as appropriate. 

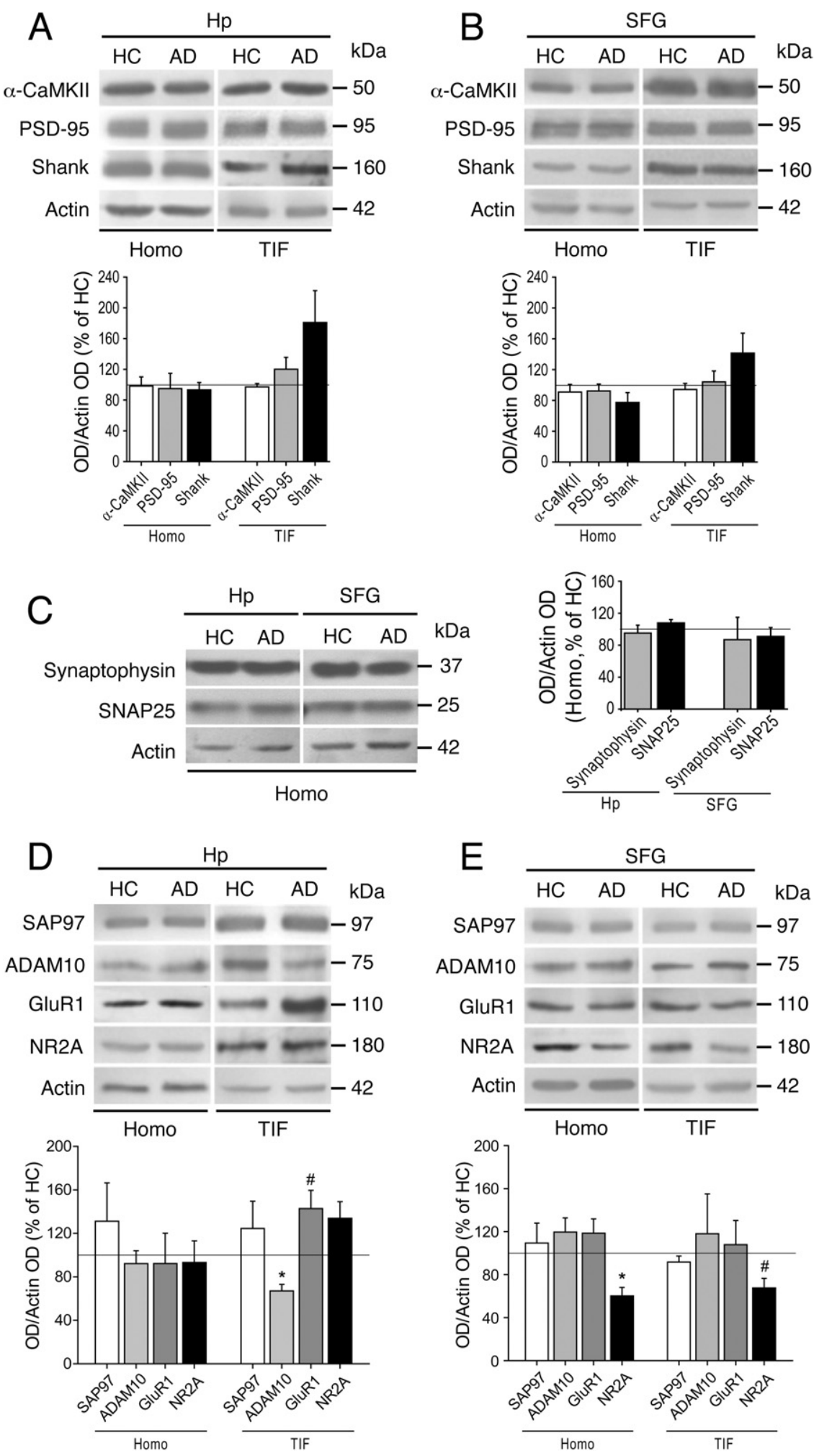

Fig. 1. Synaptic levels of A disintegrin and metalloproteinase 10 (ADAM10) and GluR1 are altered in hippocampus (Hp) of Alzheimer's disease (AD) patients. (A) Western blot (WB) of homogenate and triton insoluble fraction (TIF) obtained from Hp of AD patients and healthy controls (HC) performed with anti- $\alpha$-CaMKII, anti-PSD-95 and anti-Shank antibodies. No alterations were found in the levels of these proteins in total homogenate and in TIF. (B) WB of homogenate and TIF obtained from superior frontal gyrus (SFG) of AD patients and HC performed with anti- $\alpha$-CaMKII, anti-PSD-95 and anti-Shank antibodies. No alterations were found in the levels of these proteins in total homogenate and in TIF. (C) WB of homogenate obtained from SFG of AD patients and $\mathrm{HC}$ performed with anti-synaptophysin and anti-SNAP25 antibodies. No alterations were found in the levels of these proteins in total homogenate (D) WB of homogenate and TIF obtained from Hp of AD patients and HC performed with anti-SAP97, anti-ADAM10, anti-GluR1, and anti-NR2A antibodies. 


\section{Results}

\subsection{At Braak 4 stage of disease, synaptic structure is not affected while APP metabolism is shifted toward amyloidogenesis in Hp but not in SFG}

Although current available animal models have been extremely informative about key pathogenic pathways of $\mathrm{AD}$, to assess the relevance of SAP97 complexes with its synaptic partners in $\mathrm{AD}$ pathogenesis we exploited autoptic brain tissues obtained from 6 late onset AD patients, fulfilling criteria for Braak 4 stage, and 6 aged-matched control subjects (HC) (Table 1).

Our experimental design implied the study of the synaptic role of SAP97 in the earliest phase of AD pathogenesis, when APP metabolism is shifted toward amyloidogenesis but the loss of synaptic structure is not prominent. Thus we focused only on $\mathrm{Hp}$, the first brain area affected by the disease, and SFG, a less affected area used as negative control because no significant plaque deposition was detectable at this stage, as revealed by neuropathology reports.

To validate our experimental design and to support neuropathological data with biochemical ones, we characterized APP metabolism, postsynaptic structural composition, and presynaptic proteins in both areas of $\mathrm{HC}$ and AD patients' groups.

Firstly, in $\mathrm{Hp}$ and in SFG, we measured release of APP ectodomain generated by $\alpha$-secretase $(\operatorname{sAPP} \alpha)$ in the soluble fraction. Immunoblot experiments, performed with $6 \mathrm{E} 10$ antibody, showed a reduction in sAPP $\alpha$ levels in $\mathrm{Hp}$ of $\mathrm{AD}$ compared with $\mathrm{HC}(-38.8 \pm 12.7 \%, p=0.028$, supplementary. Fig. S1A), but not in SFG $(+33.7 \pm 56.4 \%$, $p>0.05$, supplementary Fig. S1A). To strengthen these results, APP C-terminal fragments (CTFs)-CTF99 for $\beta$-cleavage and CTF83 for $\alpha$-cleavage-were evaluated in the triton insoluble fraction (TIF), a fraction enriched in postsynaptic density proteins (Adlard et al., 2005; Kamenetz et al., 2003; Zimmermann et al., 2004). TIF proteins were separated on a $15 \%$ Tris-Tricine SDS-PAGE and immunoblot analysis was performed with 4G8 antibody. CTF83/ CTF99 ratio was significantly decreased in Hp of AD compared with $\mathrm{HC}(-25.85 \pm 8.5 \%, p=0.029$, supplementary Fig. S1B), but not in SFG $(+23.7 \pm 25.2 \%, p>0.05$, supplementary Fig. S1B).

These data confirm that, at this stage of pathogenesis, APP metabolism is shifted toward amyloidogenesis only in $\mathrm{Hp}$ and not in SFG of AD patients.

Secondly, we evaluated possible alterations in the postsynaptic structure occurring at this stage of the disease. To this, we assessed biochemically the levels and synaptic localization of the three major postsynaptic density (PSD) components, i.e., Shank, PSD-95, and $\alpha$-CaMKII (Sheng and Hoogenraad, 2007). Immunoblot experiments were performed on total homogenate and on TIF, a fraction enriched in postsynaptic density proteins. Quantitative analyses showed no significant modifications of Shank, PSD-95 and $\alpha$-CaMKII in total homogenate and TIF fraction of both Hp and SFG of AD and HC, suggesting that postsynaptic structure is preserved in our experimental groups [Hp (Homo), $\alpha$-CaMKII $=-1.8 \pm 11.9 \%$, PSD-95 $=-5.2 \pm 19.8 \%$, Shank $=-6.9 \pm 9.6 \%$; SFG (Homo), $\alpha$-CaMKII $=-9.12 \pm$ 9.8\%, PSD-95 = -7.8 $\pm 9.1 \%$, Shank $=-22.8 \pm 12.8 \%$; overall: $p>0.05$; Hp (TIF), $\alpha$-CaMKII $=-2.9 \pm 4.5 \%$, PSD-95 $=+20.8 \pm 15.5 \%$, Shank $=+80.8 \pm 41.4 \%$; SFG (TIF), $\alpha$-CaMKII $=-5.8 \pm 7.8 \%$, PSD-95 $=+3.9 \pm$ $14.1 \%$, Shank $=+41.1 \pm 25.7 \%$, overall: $p>0.05$; Fig. $1 \mathrm{~A}$ and $\mathrm{B}]$.

Moreover, we evaluated the levels of the presynaptic proteins synaptophysin and SNAP25. Quantitative analyses revealed no significant alterations of synaptophysin and SNAP25 in total homogenate of both Hp and SFG of AD and $\mathrm{HC}$, suggesting that the presynapse is still not affected in our experimental conditions $(\mathrm{Hp}$, synaptophysin = $-4.8 \pm 9.8 \%$, SNAP25 $=+7.9 \pm 4.1 \%$; SFG, synaptophysin $=-13.1 \pm 28 \%$; SNAP25 $=-9.5 \pm 11.1 \%$, overall: $p>0.05$; Fig. 1C).

\subsection{Synaptic levels of ADAM10 and GluR1, but not of $N R 2 A$, are changed in $H p$ of $A D$ patients}

We then evaluated possible alterations of synaptic localization of SAP97 and its key partners, i.e., ADAM10, GluR1, and NR2A. The levels of these proteins have been assessed in both total homogenate and TIF fraction.

SAP97 levels in both total homogenate and TIF of Hp (Homo, $+31.4 \pm 35.1 \%$; TIF, $+24.8 \pm 25.6 \%, p>0.05$; Fig. 1D) and SFG (Homo, +9.3 $\pm 18.5 \%$; TIF, $-8.5 \pm$ $5.6 \%, p>0.05$; Fig. 1E) were not affected.

Western blot analyses of TIF showed a significant reduction of ADAM10 levels in Hp of AD when compared with $\mathrm{HC}(-32.6 \pm 5.6 \%, p=0.002$, Fig. 1D) but not in SFG $(+18 \pm 37.1 \%, p>0.05$; Fig. 1E). ADAM10 levels in total homogenate were unaltered in both areas $(\mathrm{Hp},-7.7 \pm$ 11.7\%; SFG, $+19.5 \pm 13.2 \%, p>0.05$; Fig. $1 \mathrm{D}$ and E).

GluR1 levels were significantly increased in TIF membranes of $\mathrm{Hp}$ of $\mathrm{AD}$ patients when compared with $\mathrm{HC}$ subjects $(+43.0 \pm 16.6 \%, p=0.048$, Fig. 1D) but not in

\footnotetext{
No alterations were found in the levels of these proteins in total homogenate. A significant reduction of ADAM10 levels and an increase of GluR1 levels in TIF were observed in AD compared with HC (ADAM10: $* p=0.002$, GluR1: \# $p=0.048$ ). (E) WB of homogenate and TIF obtained from SFG of AD patients and HC performed with anti-SAP97, anti-ADAM10, anti-GluR1, and anti-NR2A antibodies. No alterations of SAP97, ADAM10, and GluR1 were found in the levels of these proteins in total homogenate and in TIF. NR2A was significantly reduced in both homogenate and TIF (Homo: ${ }^{*} p=0.004$, TIF: $\# p=0.015$ ). All data were normalized using actin. For all experiments quantitative analysis of immunostaining is shown as percentage of HC in the same experiment.
} 

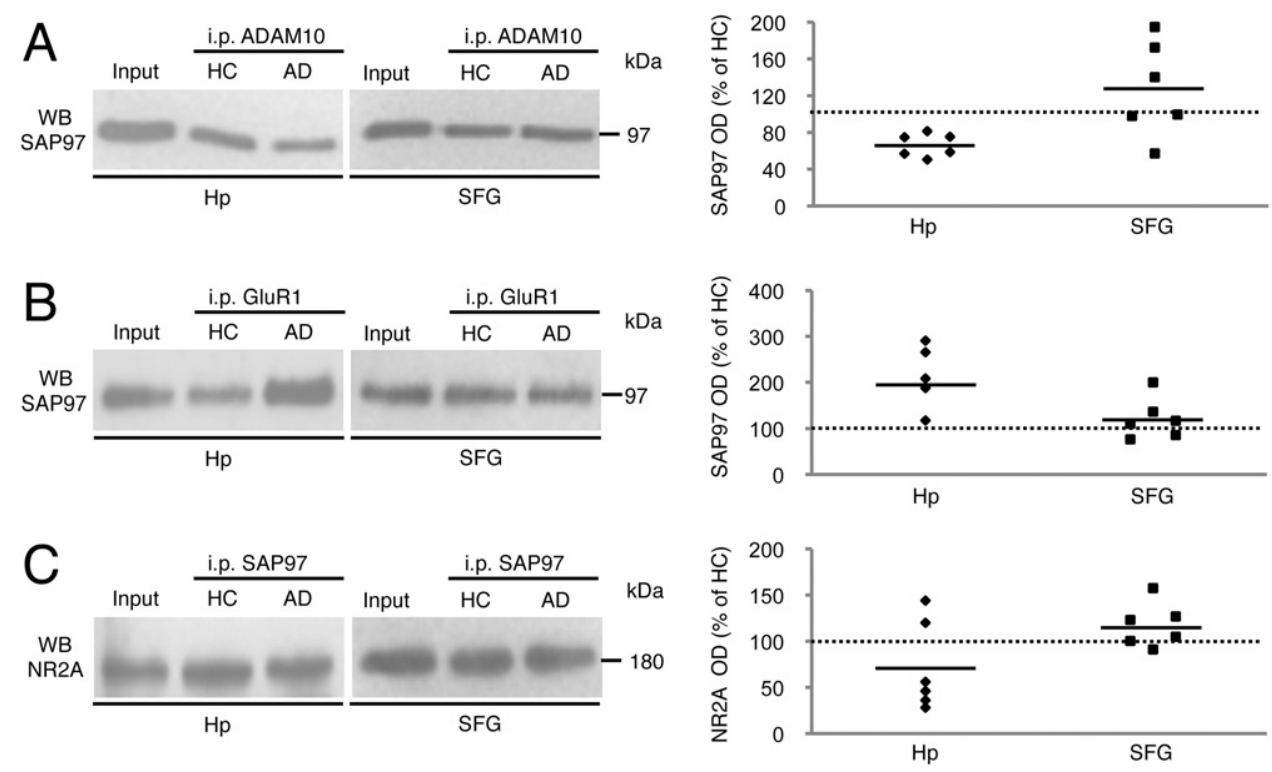

Fig. 2. SAP97 capability to associate to both A disintegrin and metalloproteinase 10 (ADAM10) and GluR1 is changed in Alzheimer disease (AD) patients' hippocampus (Hp). (A) Homogenate from Hp and superior frontal gyrus (SFG) of healthy controls (HC) and AD patients was immunoprecipitated with anti-ADAM10 antibody and SAP97 presence was evaluated. ADAM10/SAP97 association is reduced in AD patients compared with HC ( $p=0.001)$. No differences were detectable in SFG. (B) Homogenate from Hp and SFG of HC and AD patients was immunoprecipitated with anti-GluR1 antibody and SAP97 presence was evaluated. GluR1/SAP97 association is increased in AD patients compared with HC $(p=0.04)$. No differences were detectable in SFG. (C) Homogenate from Hp and SFG of HC and AD patients was immunoprecipitated with anti-SAP97 antibody and NR2A presence was evaluated. SAP97/NR2A association is not modified in AD patients compared with HC. No differences were detectable in SFG. Quantitative analysis of immunostaining is shown as percentage of $\mathrm{HC}$ in the same experiment.

SFG $(+7.9 \pm 22.4 \%, p>0.05$; Fig. 1E). Western blot analyses of homogenates revealed no alterations in GluR1 total levels both in $\mathrm{Hp}(-7.5 \pm 27.7 \%, p>0.05$, Fig. 1D) and in SFG $(+18.5 \pm 13.3 \%, p>0.05$, Fig. 1E).

Immunoblot experiments detected no significant alterations of NR2A levels both in total homogenate $(-6.6 \% \pm 19.7 \%, p$ $>0.05$, Fig. 1D) and in TIF fraction of AD Hp $(+34.1 \pm$ $15.1 \%, p>0.05$, Fig. 1E) compared with HC. In SFG of AD patients compared with $\mathrm{HC}$, NR2A levels were concomitantly reduced in both homogenate and TIF (Homo, $-39.9 \pm 8 \%, p=$ 0.004 , TIF, $-32.3 \pm 8.8 \%, p=0.015$; Fig. 1E)

These data showed an altered synaptic localization of ADAM10 and GluR1, but not of NR2A, in Hp of AD patients.

\subsection{SAP97 capability to associate to both ADAM10 and GluRl, is altered in AD patients' $H p$}

Because binding to SAP97 is pivotal for these synaptic proteins' trafficking, the ability of SAP97 to effectively bind its partners in $\mathrm{AD}$ cases and control subjects was then evaluated.

The capability of SAP97 to bind ADAM10, through the SH3 domain of SAP97, and glutamate receptor subunits, i.e., GluR1 (Leonard et al., 1998) and NR2A (Bassand et al., 1999), via its PDZ domains, was analyzed by coimmunoprecipitation experiments.

In $\mathrm{Hp}$ of AD cases compared with HC, ADAM10/SAP97 coimmunoprecipitation was reduced $(-33.7 \pm 4.9 \%, p=$ 0.001 ; Fig. 2A). No differences were detectable between the two groups in SFG $(+26.9 \pm 21.0, p>0.05$; Fig. $2 \mathrm{~A})$. Coimmunoprecipitation experiments revealed a significant increase of GluR1/SAP97 coprecipitation in Hp homogenates of $\mathrm{AD}$ patients, when compared with $\mathrm{HC}(+90.3 \pm$ $34.2 \%, p=0.04$; Fig. 2B). In the SFG of AD cases compared with $\mathrm{HC}$, no alterations were observed $(+21.1 \pm$ $18 \%, p>0.05$; Fig. 2B).

Western blot analyses of NR2A/SAP97 immunocomplex showed no significant differences between $\mathrm{AD}$ and $\mathrm{HC}$ subjects in $\mathrm{Hp}(-28.3 \pm 21.5 \%, p>0.05$; Fig. 2C). Because NR2A expression levels were reduced in SFG (Fig. 1E) coimmunoprecipitation assays were performed precipitating the unchanged protein, i.e., SAP97. Coimmunoprecipitation assays of NR2A/SAP97 complex were performed from SFG homogenates and no significant alterations were found $(+17.3 \pm 9.8 \%, p>0.05$; Fig. $2 \mathrm{C})$.

These data point to an alteration of SAP97 capability to bind ADAM10 and GluR1, but not NR2A, in AD Hp.

\subsection{SAP97 binding capacity is modified in $A D$ patients' $\mathrm{Hp}$}

To further confirm these results and to assess SAP97 binding capacity in an in vitro system, pull-down experiments were carried out. For each binding partner, fusion proteins between GST and the domains of ADAM10, GluR1, and NR2A responsible for the interaction with SAP97 were generated, purified, and incubated with either Hp or SFG homogenate of AD and HC. 

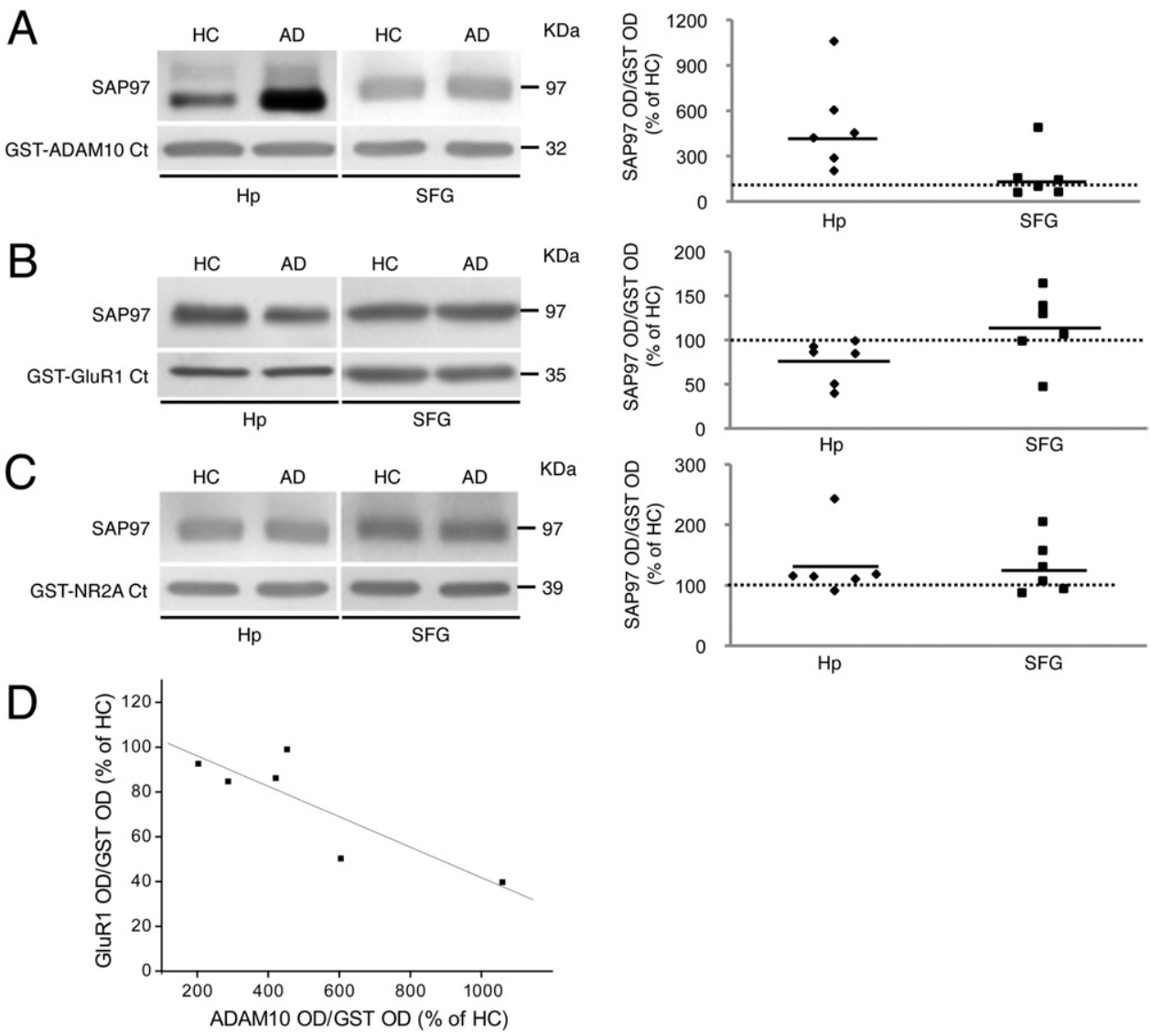

Fig. 3. SAP97 capability to pull-down A disintegrin and metalloproteinase 10 (ADAM10) and GluR1, but not NR2A, is modified in hippocampus (Hp) of Alzheimer disease (AD) patients. (A) Glutathione S-transferase (GST)-ADAM10 C-terminal tail fusion protein was incubated in a pull-down assay with total homogenate from $\mathrm{Hp}$ and superior frontal gyrus (SFG) of AD patients and healthy controls (HC). Western blot (WB) analysis was performed with anti-SAP97 antibody. SAP97 amount pulled down by GST-ADAM10 C-terminal fusion protein is significantly higher in AD Hp compared with HC $(p=0.009)$ while no alterations are detectable in SFG. (B) GST-GluR1 C-terminal tail fusion protein was incubated, in a pull-down assay, with total homogenate from Hp and SFG of AD and HC. WB analysis was performed with anti-SAP97 antibody. SAP97 pulled down by GST-GluR1 C-terminal fusion protein is reduced in AD Hp compared with HC ( $p=0.033)$ while no alterations are detectable in SFG. (C) GST-NR2A C-terminal (1349-1464) fusion protein was incubated in a pull-down assay with total homogenate from Hp and SFG of AD and HC. WB analysis was performed with anti-SAP97 antibody. No differences were detectable in both areas between AD patients and HC. (D) In Hp, the levels of SAP97 pulled down by GluR1 C-terminal tail negatively correlate to the amount of SAP97 precipitated by ADAM10 tail (correlation analysis with Pearson method: $p=0.031$ ). No correlation was present in SFG. All data were normalized on GST staining. For all experiments quantitative analysis of immunostaining is shown as percentage of HC in the same experiment.

Pull-down assays were performed and Western blot analysis revealed a band corresponding to SAP97, as expected. Quantitative analyses showed a significant increase of endogenous SAP97 binding to ADAM10 cytoplasmic tail, a significant reduction of GluR1 C-terminal tail capability to bind SAP97, and no alterations of SAP97 precipitation with GST-NR2A C-terminal tail (1349-1464) in Hp of AD patients when compared with HC (ADAM10, +404.9 \pm $124.6 \%, p=0.009$; GluR1, $-24.5 \pm 9.9 \%, p=0.033$; NR2A, $+32.5 \pm 22.5 \%, p>0.05$ Fig. $3 \mathrm{~A}-\mathrm{C}$ ).

Pull-down experiments carried out from SFG samples did not reveal any significant modification of all proteins between the two groups (ADAM10, +69.1 $\pm 66.2 \%$; GluR1 + 14.4 $\pm 16.5 \%$; NR2A, $+30.9 \pm 18.2 \%, p>0.05$; Fig. 3A-C).

Pull-down assays data mirror the results obtained by immunoprecipitation analyses. In AD patients' Hp a reduc- tion of ADAM10/SAP97 complex entails an increased availability of SAP97 to bind in vitro ADAM10 cytoplasmic tail, as demonstrated by the pull-down assay. On the other hand, in AD Hp, GluR1/SAP97 association is enhanced and consequently in vitro experiments showed a reduced binding of SAP97 to GluR1 tail.

Correlation analysis of data obtained by pull-down experiments with Pearsons method, revealed that SAP97 binding to GluR1 correlates with ADAM10 binding in $\operatorname{Hp}(p=0.03$, Fig. 3D), but not in SFG ( $p=0.336$, Fig. 3D). These results suggest an interdependence between the alterations of ADAM10 and GluR1 binding to SAP97 in Hp of AD patients.

\section{Discussion}

Although the etiology of AD remains unclear, the leading hypothesis is that accumulation of $\mathrm{A} \beta$ peptide is the 
causative event of the pathogenesis (Hardy and Selkoe, 2002). Nevertheless, studies in patients failed to demonstrate a clear correlation between $\mathrm{A} \beta$ plaques and cognitive impairment. On a different ground, a higher correlation has been shown between the loss of synapses and decreased cognitive function (Terry et al., 1991). Lately, this led to the emerging concept that $\mathrm{AD}$ pathogenesis has to be considered the result of a complex interplay of crossing pathways involving amyloid cascade and synaptic dysfunction (Bossy-Wetzel et al., 2004). The complete comprehension of the mechanism(s) through which these two main components of $\mathrm{AD}$ pathogenesis reciprocally interact and influence each other turned out as more and more important to reach a complete picture of the disease's molecular pathogenesis.

In the last few years, several studies aimed at understanding precisely how $\mathrm{A} \beta$ accumulation and assembly compromise synaptic structure and function of excitatory synapses, taking advantage of synthetic or cell-derived $\mathrm{A} \beta$ aggregates. This has become the centerpiece of therapeutically oriented research on the disease (Selkoe, 2008). Several studies hypothesized a direct action of $\mathrm{A} \beta$ peptides and oligomers on the postsynaptic membrane of excitatory synapse, describing in in vitro systems an interference of $A \beta$ on glutamate receptor trafficking and function and on spine formation (Lacor et al., 2007; Shankar et al., 2007, 2008; Snyder et al., 2005). However, cellular and molecular targets of $\mathrm{A} \beta$ and/or early molecular events initiating the process of synaptic failure are still unknown.

In this study, we changed perspective and tried to address the question by studying possible intraneuronal pathways bridging synaptic function and amyloid production. We focused on SAP97, a cargo protein responsible not only for the trafficking of ionotropic glutamate receptor subunits, i.e., GluR1 (Leonard et al., 1998) and NR2A (Bassand et al., 1999), but also for the synaptic localization of $\alpha$-secretase ADAM10 (Marcello et al., 2007). Therefore, SAP97 is involved in both functional organization of the glutamatergic synapse (Gardoni et al., 2008; Garner et al., 2000) and modulation of $\alpha$-secretase activity (Marcello et al., 2007). Notably a dysfunction of SAP97 has been already reported in AD patients brain (Wakabayashi et al., 1999), as well as in other neurological disorders (Nash et al., 2005; Sato et al., 2008).

The aim of our study was to investigate possible alterations of SAP97 complexes with its partners in the earlier phases of AD pathogenesis, when APP metabolism is shifted toward amyloidogenesis but the loss of synaptic structure is not prominent. To this, we exploited autoptic Hp and SFG, respectively as an affected and a less affected area, obtained from late onset $\mathrm{AD}$ patients, fulfilling criteria for Braak 4 stage, and age-matched control subjects.

We precisely defined our experimental design because results in the literature describing alterations of the glutamatergic synapses of $\mathrm{AD}$ patients at different stages of the disease are unfortunately very variable (Gylys et al., 2004;
Masliah et al., 2001; Mukaetova-Ladinska et al., 2000). At present our knowledge is a mosaic of several different results, not always obtained in comparable experimental conditions.

In light of these considerations, first of all we characterized APP metabolism and synaptic structural composition. As confirmed by the measurements of the amyloid cascade products, at this stage of disease APP metabolism is shifted toward amyloidogenesis only in Hp and not in SFG. In our experimental conditions, biochemical analyses of both presynaptic proteins and the major PSD components demonstrated that the presynapse and the postsynaptic structure are still not affected in both areas.

Here we showed an alteration of SAP97 capability to interact with its binding partners just in vulnerable brain areas of AD patients at initial stages, thus suggesting SAP97 cargo dysfunction as an early mechanism of pathogenesis.

In Hp of AD patients, levels of ADAM10 and GluR1 at synaptic membranes are respectively reduced and augmented, while NR2A localization is not affected. These alterations are specific because no modifications were found in SFG.

Important insights may be gleaned from our findings, adding new pieces to the puzzle in understanding how $\mathrm{A} \beta$ formation is upregulated in sporadic AD. Our results suggest that at this stage of the disease the reduction of $\alpha$-secretase activity could be ascribed to a defect in ADAM10 trafficking rather than to an alteration of its expression. Indeed, it turned out that $\alpha$-secretase activity is reduced only in $\mathrm{Hp}$ of $\mathrm{AD}$ patients, the brain area where ADAM10 localization at postsynaptic membranes is impaired.

Furthermore, membrane protein trafficking has a pivotal role not only in the process leading to $\mathrm{A} \beta$ formation, but also in the mechanisms regulating synaptic function. Indeed, molecular memory and learning-related synaptic plasticity take advantage of receptor trafficking, insertion and removal to finely modulate both long term maintenance of synaptic transmission and acute changes in synaptic strength (Newpher and Ehlers, 2008). In particular, changes in AMPA receptor levels have been implicated in the expression and maintenance of activity-dependent synaptic plasticity phenomena, i.e., long term potentiation (LTP) and long term depression (LTD) (Bredt and Nicoll, 2003; Collingridge et al., 2004). Therefore, the increased localization of GluR1 at the synaptic site in AD Hp could reflect an impairment of dynamic regulation of the number of synaptic receptors, thus probably being involved in the pathogenic processes responsible for synaptic dysfunction.

The altered localization of ADAM10 and GluR1 could be ascribed to a failure of SAP97 to properly bind these partners. Both immunoprecipitation and pull-down assays demonstrated that SAP97 failed to correctly couple to ADAM10 and GluR1, but not to NR2A, in AD Hp.

The observations that ADAM10/SAP97 complex is markedly reduced and that GluR1/SAP97 interaction is sig- 
nificantly increased in $\mathrm{Hp}$ of $\mathrm{AD}$ patients indicates alterations of SAP97 as a key element of molecular pathogenesis of the disease. Indeed, SAP97 has been previously described as a determinant for the activity of the $\alpha$-secretase enzyme ADAM10. A disruption of ADAM10/SAP97 complex in rodents led to a reduction of ADAM10 localization at postsynaptic sites and to a shift of APP metabolism toward the amyloidogenic pathway (Marcello et al., 2007). Moreover, GluR1 interactions seem to dominate the regulation of exocytosis during long term potentiation (Shepherd and Huganir, 2007): the PDZ ligand on GluR1 appears critical for activity-dependent trafficking of AMPA receptor surface, and expression (Piccini and Malinow, 2002) and inducible exocytosis are impaired when the C-terminus is mutated (Passafaro et al., 2001).

In addition, the altered binding affinity to cytoplasmic tails of ADAM10 and GluR1 in AD Hp is strictly interdependent. This negative correlation suggests that a modification of SAP97 function in AD pathogenesis could lead to a reduced ADAM10 delivery to the postsynaptic membrane, thus shifting APP metabolism toward amyloidogenesis, and to an increased localization of GluR1 which impairs synaptic function.

In conclusion, this study sheds light on an open question in $\mathrm{AD}$ research concerning the mutual interaction between different pathways of pathogenesis. In particular, we depict a new mechanism of pathogenesis which goes beyond both amyloid production/release in the extracellular fluids and deleterious effects of $A \beta$ on neighboring cells, but rather points to a dysfunction of a key intracellular process: local protein trafficking and synapse assembly.

This new vision may help not only in indicating SAP97 as a point of convergence between amyloid cascade and synaptic function, but also in a different interpretation of $\mathrm{AD}$ which in this new context can be perceived as synaptic trafficking defect pathology.

\section{Disclosure statement}

All authors report no biomedical financial interests or potential conflicts of interest.

All procedures were in accordance with the National Institutes of Health (NIH) Guide for the Care and Use of Laboratory human tissue and were approved by the Ethics Committee of the University of Milan, Italy.

\section{Acknowledgements}

The brain tissues were obtained from the Netherlands Brain Bank, Netherlands Institute for Neuroscience, Amsterdam. We thank A. Longhi and E. Zianni for technical assistance, and S. Tadini and G. Rigillo for excellent practical work. The research leading to these results has received funding from the European Union's Seventh Framework Programme (FP7 2007-2013) under Grant Agreement $n^{\circ}$ PIAP-GA-2008-217902. This work was supported by CARIPLO (2008) to MDL and PRIN \# 20079E5Y3E to AP.

\section{Appendix A. Supplementary data}

Supplementary data associated with this article can be found, in the online version, at doi:10.1016/j.neurobiolaging. 2010.09.015.

\section{References}

Adlard, P.A., Perreau, V.M., Pop, V., Cotman, C.W., 2005. Voluntary exercise decreases amyloid load in a transgenic model of Alzheimer's disease. J. Neurosci. 25, 4217-4221.

Anders, A., Gilbert, S., Garten, W., Postina, R., Fahrenholz, F., 2001. Regulation of the alpha-secretase ADAM10 by its prodomain and proprotein convertases. FASEB J. 15, 1837-1839.

Bassand, P., Bernard, A., Rafiki, A., Gayet, D., Khrestchatisky, M., 1999. Differential interaction of the tSXV motifs of the NR1 and NR2A NMDA receptor subunits with PSD-95 and SAP97. Eur. J. Neurosci. 11, 2031-2043.

Bertoni-Freddari, C., Fattoretti, P., Casoli, T., Caselli, U., Meier-Ruge, W., 1996. Deterioration threshold of synaptic morphology in aging and senile dementia of Alzheimer's type. Anal. Quant. Cytol. Histol. 18, 209-213.

Bertoni-Freddari, C., Fattoretti, P., Solazzi, M., Giorgetti, B., Di Stefano, G., Casoli, T., Meier-Ruge, W., 2003. Neuronal death versus synaptic pathology in Alzheimer's disease. Ann. N. Y. Acad. Sci. 1010, 635638.

Bossy-Wetzel, E., Schwarzenbacher, R., Lipton, S.A., 2004. Molecular pathways to neurodegeneration. Nat. Med. 10, S2-S9.

Braak, H., Braak, E., 1991. Neuropathological staging of Alzheimer-related changes. Acta Neuropathol. 82, 239-259.

Bredt, D.S., Nicoll, R.A., 2003. AMPA receptor trafficking at excitatory synapses. Neuron 40, 361-379.

Collingridge, G.L., Isaac, J.T., Wang, Y.T., 2004. Receptor trafficking and synaptic plasticity. Nat. Rev. Neurosci. 5, 952-962.

DeKosky, S.T., Scheff, S.W., 1990. Synapse loss in frontal cortex biopsies in Alzheimer's disease: correlation with cognitive severity. Ann. Neurol. 27, 457-464.

Elias, G.M., Nicoll, R.A., 2007. Synaptic trafficking of glutamate receptors by MAGUK scaffolding proteins. Trends Cell Biol. 17, 343-352.

Gardoni, F., Marcello, E., Di Luca, M., 2008. Postsynaptic density-membrane associated guanylate kinase proteins (PSD-MAGUKs) and their role in CNS disorders. Neuroscience 158, 324-33.

Gardoni, F., Bellone, C., Cattabeni, F., Di Luca, M., 2001. Protein kinase $\mathrm{C}$ activation modulates alpha-calmodulin kinase II binding to NR2A subunit of $N$-methyl-D-aspartate receptor complex. J. Biol. Chem. 276, 7609-7613.

Gardoni, F., Schrama, L.H., van Dalen, J.J., Gispen, W.H., Cattabeni, F., Di Luca, M., 1999. AlphaCaMKII binding to the C-terminal tail of NMDA receptor subunit NR2A and its modulation by autophosphorylation. FEBS Lett. 456, 394-398.

Gardoni, F., Mauceri, D., Fiorentini, C., Bellone, C., Missale, C., Cattabeni, F., Di Luca, M., 2003. CaMKII-dependent phosphorylation regulates SAP97/NR2A interaction. J. Biol. Chem. 278, 44745-44752.

Garner, C.C., Nash, J., Huganir, R.L., 2000. PDZ domains in synapse assembly and signalling. Trends Cell Biol. 10, 274-280.

Gylys, K.H., Fein, J.A., Yang, F., Wiley, D.J., Miller, C.A., Cole, G.M., 2004. Synaptic changes in Alzheimer's disease: increased amyloid-beta and gliosis in surviving terminals is accompanied by decreased PSD-95 fluorescence. Am. J. Pathol. 165, 1809-1817.

Hardy, J., Selkoe, D.J., 2002. The amyloid hypothesis of Alzheimer's disease: progress and problems on the road to therapeutics. Science 297, 353-356. 
Kamenetz, F., Tomita, T., Hsieh, H., Seabrook, G., Borchelt, D., Iwatsubo, T., Sisodia, S., Malinow, R., 2003. APP processing and synaptic function. Neuron 37, 925-937.

Kim, E., Sheng, M., 2004. PDZ domain proteins of synapses. Nat. Rev. Neurosci. 5, 771-781.

Lacor, P.N., Buniel, M.C., Furlow, P.W., Clemente, A.S., Velasco, P.T., Wood, M., Viola, K.L., Klein, W.L., 2007. Abeta oligomer-induced aberrations in synapse composition, shape, and density provide a molecular basis for loss of connectivity in Alzheimer's disease. J. Neurosci. 27, 796-807.

Lammich, S., Kojro, E., Postina, R., Gilbert, S., Pfeiffer, R., Jasionowski, M., Haass, C., Fahrenholz, F., 1999. Constitutive and regulated alphasecretase cleavage of Alzheimer's amyloid precursor protein by a disintegrin metalloprotease. Proc. Natl. Acad. Sci. U. S. A. 96, 3922 3927.

Leonard, A.S., Davare, M.A., Horne, M.C., Garner, C.C., Hell, J.W., 1998. SAP97 is associated with the alpha-amino-3-hydroxy-5-methylisoxazole-4-propionic acid receptor GluR1 subunit. J. Biol. Chem. 273, 19518-19524.

Marcello, E., Gardoni, F., Mauceri, D., Romorini, S., Jeromin, A., Epis, R., Borroni, B., Cattabeni, F., Sala, C., Padovani, A., Di Luca, M., 2007. Synapse-associated protein-97 mediates alpha-secretase ADAM10 trafficking and promotes its activity. J. Neurosci. 27, 1682-1691.

Masliah, E., Mallory, M., Alford, M., DeTeresa, R., Hansen, L.A., McKeel, D.W., Jr, Morris, J.C., 2001. Altered expression of synaptic proteins occurs early during progression of Alzheimer's disease. Neurology 56, $127-129$.

Mauceri, D., Gardoni, F., Marcello, E., Di Luca, M., 2007. Dual role of CaMKII-dependent SAP97 phosphorylation in mediating trafficking and insertion of NMDA receptor subunit NR2A. J. Neurochem. 100, 1032-1046.

Mukaetova-Ladinska, E.B., Garcia-Siera, F., Hurt, J., Gertz, H.J., Xuereb, J.H., Hills, R., Brayne, C., Huppert, F.A., Paykel, E.S., McGee, M., Jakes, R., Honer, W.G., Harrington, C.R., Wischik, C.M., 2000. Staging of cytoskeletal and beta-amyloid changes in human isocortex reveals biphasic synaptic protein response during progression of Alzheimer's disease. Am. J. Pathol. 157, 623-636.

Nakagawa, T., Futai, K., Lashuel, H.A., Lo, I., Okamoto, K., Walz, T., Hayashi, Y., Sheng, M., 2004. Quaternary structure, protein dynamics, and synaptic function of SAP97 controlled by L27 domain interactions. Neuron 44, 453-467.

Nash, J.E., Johnston, T.H., Collingridge, G.L., Garner, C.C., Brotchie, J.M., 2005. Subcellular redistribution of the synapse-associated proteins PSD-95 and SAP97 in animal models of Parkinson's disease and L-DOPA-induced dyskinesia. FASEB J. 19, 583-585.

Newpher, T.M., Ehlers, M.D., 2008. Glutamate receptor dynamics in dendritic microdomains. Neuron 58, 472-497.

Passafaro, M., Piech, V., Sheng, M., 2001. Subunit-specific temporal and spatial patterns of AMPA receptor exocytosis in hippocampal neurons. Nat. Neurosci. 4, 917-926.

Piccini, A., Malinow, R., 2002. Critical postsynaptic density 95/disc large/ zonula occludens-1 interactions by glutamate receptor 1 (GluR1) and GluR2 required at different subcellular sites. J. Neurosci. 22, 53875392.

Postina, R., Schroeder, A., Dewachter, I., Bohl, J., Schmitt, U., Kojro, E., Prinzen, C., Endres, K., Hiemke, C., Blessing, M., Flamez, P., Dequenne, A., Godaux, E., van Leuven, F., Fahrenholz, F., 2004. A disintegrin-metalloproteinase prevents amyloid plaque formation and hippocampal defects in an Alzheimer disease mouse model. J. Clin. Invest. 113, 1456-1464.

Reddy, P.H., Mani, G., Park, B.S., Jacques, J., Murdoch, G., Whetsell, W., Jr, Kaye, J., Manczak, M., 2005. Differential loss of synaptic proteins in Alzheimer's disease: implications for synaptic dysfunction. J. Alzheimers Dis. 7, 103-117, Discussion, 173-180.

Sans, N., Racca, C., Petralia, R.S., Wang, Y.X., McCallum, J., Wenthold, R.J., 2001. Synapse-associated protein 97 selectively associates with a subset of AMPA receptors early in their biosynthetic pathway. J. Neurosci. 21, 7506-7516.

Sato, J., Shimazu, D., Yamamoto, N., Nishikawa, T., 2008. An association analysis of synapse-associated protein. 97 (SAP97) gene in schizophrenia. J. Neural Transm. 115, 1355-1365.

Scheff, S.W., Price, D.A., Schmitt, F.A., DeKosky, S.T., Mufson, E.J., 2007. Synaptic alterations in CA1 in mild Alzheimer disease and mild cognitive impairment. Neurology 68, 1501-1508.

Schluter, O.M., Xu, W., Malenka, R.C., 2006. Alternative N-terminal domains of PSD-95 and SAP97 govern activity-dependent regulation of synaptic AMPA receptor function. Neuron 51, 99-111.

Selkoe, D.J., 2008. Soluble oligomers of the amyloid beta-protein impair synaptic plasticity and behavior. Behav. Brain Res. 192, 106-113.

Shankar, G.M., Bloodgood, B.L., Townsend, M., Walsh, D.M., Selkoe, D.J., Sabatini, B.L., 2007. Natural oligomers of the Alzheimer amyloid-beta protein induce reversible synapse loss by modulating an NMDA-type glutamate receptor-dependent signaling pathway. J. Neurosci. 27, 2866-2875.

Shankar, G.M., Li, S., Mehta, T.H., Garcia-Munoz, A., Shepardson, N.E., Smith, I., Brett, F.M., Farrell, M.A., Rowan, M.J., Lemere, C.A., Regan, C.M., Walsh, D.M., Sabatini, B.L., Selkoe, D.J., 2008. Amyloid-beta protein dimers isolated directly from Alzheimer's brains impair synaptic plasticity and memory. Nat. Med. 14, 837-842.

Sheng, M., Hoogenraad, C.C., 2007. The postsynaptic architecture of excitatory synapses: a more quantitative view. Annu. Rev. Biochem. $76,823-847$.

Shepherd, J.D., Huganir, R.L., 2007. The cell biology of synaptic plasticity: AMPA receptor trafficking. Annu. Rev. Cell Dev. Biol. 23, 613643.

Snyder, E.M., Nong, Y., Almeida, C.G., Paul, S., Moran, T., Choi, E.Y., Nairn, A.C., Salter, M.W., Lombroso, P.J., Gouras, G.K., Greengard, P., 2005. Regulation of NMDA receptor trafficking by amyloid-beta. Nat. Neurosci. 8, 1051-1058.

Terry, R.D., Masliah, E., Salmon, D.P., Butters, N., DeTeresa, R., Hill, R., Hansen, L.A., Katzman, R., 1991. Physical basis of cognitive alterations in Alzheimer's disease: synapse loss is the major correlate of cognitive impairment. Ann. Neurol. 30, 572-580.

Vassar, R., 2001. The beta-secretase, BACE: a prime drug target for Alzheimer's disease. J. Mol. Neurosci. 17, 157-170.

Wakabayashi, K., Narisawa-Saito, M., Iwakura, Y., Arai, T., Ikeda, K., Takahashi, H., Nawa, H., 1999. Phenotypic down-regulation of glutamate receptor subunit GluR1 in Alzheimer's disease. Neurobiol. Aging 20, 287-295.

Zimmermann, M., Gardoni, F., Marcello, E., Colciaghi, F., Borroni, B., Padovani, A., Cattabeni, F., Di Luca, M., 2004. Acetylcholinesterase inhibitors increase ADAM10 activity by promoting its trafficking in neuroblastoma cell lines. J. Neurochem. 90, 1489-1499. 